\title{
A NEW RESULT ON THE CONVERGENCE OF NONHOMOGENEOUS STOCHASTIC CHAINS
}

\author{
BY
}

ARUNAVA MUKHERJEA

\begin{abstract}
AbstrACr. Nonhomogeneous stochastic chains with a finite number of states are considered in this paper. Convergence of such chains is established here in terms of strong ergodicity of certain related chains of smaller size. These results are shown to be best possible and extend earlier results of Maksimov. Nonnegative idempotent matrices are also considered.
\end{abstract}

1. Introduction. There are many papers, recently and earlier, which considered various problems concerning nonhomogeneous stochastic chains. Some of the better known contributors in this area are Dobrushin, Hajnal, Maksimov, Sarymsakov and Wolfowitz. For some of the references of these authors and other extensive works in this area, we refer the reader to [3], [7]. For some historical comments on Doeblin's work in this area in the context of weak ergodicity, we refer to [8].

Our paper here is mostly concerned with an interesting theorem of Maksimov in [4]. Maksimov considers nonhomogeneous bistochastic chains with a finite number of states and establishes the convergence of such chains in terms of strong ergodicity of certain chains of smaller size. To be more specific, let $\left(P_{n}\right)$ be a sequence of $s \times s$ bistochastic matrices (i.e. matrices with nonnegative entries adding up to 1 in each row and column separately) such that for each $k$, the sequence $P_{k, n} \equiv P_{k+1} P_{k+2} \ldots P_{n}$ converges to some bistochastic matrix $B_{k}$. Then it is easy to verify that the sequence $\left(B_{k}\right)$ converges to a bistochastic idempotent matrix $B_{\infty}$. It is well known (and can be proven easily, see, for instance, Maksimov's paper) that for every such bistochastic idempotent matrix $B_{\infty}$, there is a basis, that is, a partition of the set $\{1,2, \ldots, s\}$ into disjoint subsets $C_{1}, C_{2}, \ldots, C_{p}$ such that

$$
\begin{aligned}
\left(B_{\infty}\right)_{i j} & =0 \quad \text { for } i \in C_{k_{1}}, j \in C_{k_{2}}\left(k_{1} \neq k_{2}\right), \\
& =\frac{1}{n\left(C_{k}\right)} \quad \text { if } i \in C_{k}, j \in C_{k},
\end{aligned}
$$

where $n\left(C_{k}\right)=$ the number of elements in $C_{k}$. The most interesting (and perhaps the deepest) result of Maksimov is then that for $i$ and $j$ belonging to two different $C_{k}$ 's,

Received by the editors July 6, 1979.

1980 Mathematics Subject Classification. Primary 60B10, 60J10.

Key words and phrases. Nonhomogeneous Markov chain, basis, stochastic matrix, idempotent matrix, probability measures on semigroups, nonnegative matrix. 


$$
\sum_{n=1}^{\infty} P_{i j}^{(n)}<\infty
$$

where $P_{n} \equiv\left(p_{i j}^{(n)}\right)$. He uses this result to conclude that for a sequence $\left(P_{n}\right)$ of bistochastic matrices, the products $P_{k, n}$ converge as $n \rightarrow \infty$ for every $k$ with a basis $\left\{C_{1}, C_{2}, \ldots, C_{p}\right\}$ iff (i) $\sum_{n=1}^{\infty} p_{i j}^{(n)}$ is convergent for $i$ and $j$ belonging to any two different $C_{k}$ 's and (ii) for each $i, 1 \leqslant i \leqslant p$, the stochastic chain $\left(Q_{n}^{(i)}\right)$ is strongly ergodic, where $Q_{n}^{(i)}$ is an $n\left(C_{i}\right) \times n\left(C_{i}\right)$ stochastic matrix obtained from $P_{n}$ in the following manner: for $j$ and $t$ in $C_{i},\left(Q_{n}^{(i)}\right)_{j t}=p_{j t}^{(n)} / \Sigma_{k \in C_{i}} p_{j k}^{(n)}$. Maksimov's method in proving the above (and other) results was mainly based upon his earlier results on convolution products of probability measures on finite groups. For these kinds of algebraic results, his methods, though unorthodox and new, are effective. We will show, however, that his method of correspondence between bistochastic matrices and measures on finite groups, though admittedly convenient, can avoid the use of his prior results on groups and thus can be simplified. But the main purpose of our paper is not simplifying his proofs. Our main aim is to show that Maksimov-type results hold for a larger class of stochastic chains (which contain properly and is, in fact, much larger than, the class of bistochastic chains). Our result (Theorem 8) is also best possible. Maksimov's results on groups are not applicable or useful for our purpose. Naturally our method is completely different from Maksimov's, though we will use Maksimov-type correspondences extended for stochastic matrices to serve our purpose. Our main results are Theorems 7 and 8. We also give a characterization of the ergodic classes of a convergent nonhomogeneous chain. In $\$ 4$, we consider an application. In the last section, we consider nonnegative (rather than stochastic) idempotent matrices and present some basic results on their structures.

\section{Preliminaries.}

Definition 1. Let $P$ be an $s \times s$ stochastic matrix and $S$ be the multiplicative semigroup of $s \times s$ stochastic 0-1 matrices (i.e. matrices where the entries are either 0 or 1). Suppose that $P=\sum_{i=1}^{n} a_{i} P_{i}$, where $\left\{a_{1}, a_{2}, \ldots, a_{n}\right\}$ are nonnegative reals with sum 1 and $P_{i}$ 's are 0 -1 matrices in $S$. If $\mu$ is the probability measure on $S$ such that $\mu\left(P_{i}\right)=a_{i}, 1 \leqslant i \leqslant n$, then we define $P \leftrightarrow \mu$.

We will often say that $\mu$ represents $P$ if $P \leftrightarrow \mu$. This representation is, fortunately or unfortunately, not unique. However, for every stochastic matrix, there is at least one probability measure representing it. (See [6] for proofs of these facts.) Also, if $\mu$ and $\lambda$ are two probability measures on $S$ (as defined above) such that $P \leftrightarrow \mu$ and $Q \leftrightarrow \lambda$, then it is easily verified that the usual convolution product $\mu \cdot \lambda$ defined by

$$
\mu \cdot \lambda(x)=\sum_{z y=x} \mu(z) \lambda(y)
$$

is a representation for the matrix product $P Q$. Let $P(S)$ denote the set of all probability measures on $S$. Then, if $\left(P_{n}\right)$ is a sequence of stochastic matrices and $\left(\mu_{n}\right)$ is a sequence in $P(S)$ such that for each $n, P_{n} \leftrightarrow \mu_{n}$, then the weak convergence of the convolution products $\mu_{k, n}=\mu_{k+1} \mu_{k+2} \ldots \mu_{n}$ as $n$ tends to $\infty$ (here weak convergence is the same as pointwise convergence since $S$ is finite) implies 
the convergence of the matrix products $P_{k, n}$. The converse is not true. For example, let $s=2$ and the sequence $P_{n}$ be given by

$$
P_{n}=\left(\begin{array}{ll}
a(n) & b(n) \\
a(n) & b(n)
\end{array}\right), \quad \text { where } a(n)=\frac{1}{2}+\frac{2}{n} \text { and } b(n)=\frac{1}{2}-\frac{2}{n} .
$$

Let $c_{n}$ be a nonconvergent sequence of positive reals such that each $c_{n}<\frac{1}{8}$. Define the measures $\mu_{n}$ by prescribing the values $\frac{1}{4}-c_{n}-1 / n$ to each of $\left(\begin{array}{ll}0 & 1 \\ 1 & 0\end{array}\right)$ and $\left(\begin{array}{ll}1 & 9 \\ 0 & 1\end{array}\right)$, $\frac{1}{4}+c_{n}-1 / n$ to $\left(\begin{array}{ll}0 & 1 \\ 0 & 1\end{array}\right)$, and $\frac{1}{4}+c_{n}+3 / n$ to $\left(\begin{array}{ll}1 & 0 \\ 1 & 0\end{array}\right)$. Then, $P_{n} \leftrightarrow \mu_{n}$. Though $P_{n}$ 's converge, it is clear that $\mu_{n}$ 's do not.

Though we do not deal with infinite dimensional matrices in this paper, we will point out the difficulties involved in the infinite situation. In that spirit, our Lemma 1 describes how and in what form the above "finite dimensional" statements can be made in the infinite case. First note that an infinite stochastic matrix $D$ where the $n$th row entries are

$$
(1 / n, 1 / n, \ldots, 1 / n, 0,0, \ldots)
$$

is not a convex combination of infinite $0-1$ stochastic matrices. The reason is: if $\sum_{i} a_{i} P_{i}$, where $P_{i}$ 's are infinite 0 -1 stochastic matrices and $a_{i}$ 's are positive reals with sum 1 , equals $D$, then every row of $D$ must contain at least one entry greater than or equal to $a_{1}$, and this is not possible. Similar questions in the context of infinite doubly stochastic matrices have been studied in detail earlier; see [6].

LEMMA 1. Let $\left(P_{n}\right)$ be a sequence of countably infinite stochastic matrices such that each $P_{n}$ is a finite (or countably infinite) convex combination of 0-1 stochastic matrices. Suppose that $P_{n} \leftrightarrow \mu_{n}$, where $\mu_{n}$ 's are probability measures on the countable discrete semigroup $S$ generated by all these $0-1$ stochastic matrices. If $\mu_{n} \rightarrow \mu$ weakly for some $\mu \in P(S)$, then

$$
\left\|P_{n}-P\right\| \equiv \sup _{i} \sum_{j=1}^{\infty}\left|\left(P_{n}\right)_{i j}-P_{i j}\right|
$$

tends to zero as $n \rightarrow \infty$, where $P \leftrightarrow \mu$.

Proof. Given $\varepsilon>0$, there is a finite subset $A \subset S$ such that for some $N, n \geqslant N$

(i) $\mu_{n}(A)>1-\varepsilon$,

(ii) $\mu(A)>1-\varepsilon$,

(iii) $\Sigma_{x \in A}\left|\mu_{n}(x)-\mu(x)\right|<\varepsilon$.

Now by definition, $P_{n}=\Sigma_{x \in S} x \mu_{n}(x)$ and $P=\Sigma_{x \in S} x \mu(x)$. Let $B_{i j}=\{x \in S$ : $\left.x_{i j}=1\right\}$. Then for $j \neq k, B_{i j} \cap B_{i k}=\varnothing(\forall i)$. Thus, we can write: for any $i$ and $n \geqslant N$,

$$
\begin{aligned}
\sum_{j=1}^{\infty}\left|\left(P_{n}\right)_{i j}-P_{i j}\right| & \leqslant \sum_{j=1}^{\infty} \sum_{x \in B_{i j}}\left|\mu_{n}(x)-\mu(x)\right| \\
& \leqslant \sum_{j=1}^{\infty} \sum_{x \in B_{i j} \cap A}\left|\mu_{n}(x)-\mu(x)\right|+\sum_{j=1}^{\infty} \sum_{x \in B_{i j} \cap(S-A)}\left[\mu_{n}(x)+\mu(x)\right] \\
& \leqslant \sum_{x \in A}\left|\mu_{n}(x)-\mu(x)\right|+\mu_{n}(S-A)+\mu(S-A) \leqslant 3 \varepsilon .
\end{aligned}
$$

The lemma is now clear. 
Note that the (infinite case) converse of the situation described in Lemma 1 does not hold. An example similar to the one (preceding the lemma) in the finite case can be easily constructed. In the infinite case even if $\left\|P_{n}-P\right\| \rightarrow 0$ as $n \rightarrow \infty$, it is not clear if there will be a weak limit point for a sequence of probability measures $\mu_{n}$ on $S$ (where $\mu_{n} \leftrightarrow P_{n}$ and $S$ is as in the lemma). Later, it will be clear that this is one reason why the extension of our results to the infinite dimensional case seems difficult.

From here on, all matrices in this paper are $s \times s$ stochastic matrices (except in $\S 5$, where we consider nonnegative matrices which are not necessarily stochastic). Let $\left(P_{n}\right)$ be a squence of matrices such that for every positive integer $k$, the sequence $P_{k, n} \equiv P_{k+1} P_{k+2} \ldots P_{n} \rightarrow Q_{k}$. Such a sequence $\left(P_{n}\right)$ will be called a convergent stochastic chain. Let $Q^{\prime}$ and $Q^{\prime \prime}$ be any two limit points of the sequence $\left\{Q_{k}: k \geqslant 1\right\}$. Then since for $k<n<m, P_{k, n} P_{n, m}=P_{k, m}$, we have as $m \rightarrow \infty$, $P_{k, n} Q_{n}=Q_{k}$ and then passing $n$ to infinity for each $k, Q_{k} Q^{\prime}=Q_{k}, Q_{k} Q^{\prime \prime}=Q_{k}$ so that

$$
Q^{\prime} Q^{\prime \prime}=Q^{\prime}
$$

This equation implies that every limit point of the $Q_{k}$ 's is an idempotent stochastic matrix. [Note that if the $P_{n}$ 's are bistochastic, then $\lim _{k \rightarrow \infty} Q_{k}$ exists, but, in the stochastic case, this is no longer true. This follows from the discussion following Lemma 2.]

Now we recall an important theorem of Doob on the structure of an idempotent stochastic matrix $B \equiv\left(B_{i j}\right)$; see [1, p. 121]. For a more general theorem, see our Theorem 11 in $\$ 5$.

LEMMA 2. There exists a partition of the set $\{1,2, \ldots, s\}$ into disjoint classes $\left\{T, C_{1}, C_{2}, \ldots, C_{p}\right\}$ such that $\{1,2, \ldots, s\}=T \cup C_{1} \cup \ldots \cup C_{p}$ and the following are true:

(i) $B_{i j}=0$ if $j \in T$;

(ii) $B_{i j}=B_{j}(>0)$ if $i, j$ both belong to the same $C_{k}$ for some $k$, $=0$ if $i, j$ belong to two different $C_{k}$ 's;

(iii) $B_{i j}=B_{j}\left(\Sigma_{k \in C_{t}} B_{i k}\right)$ if $i \in T, j \in C_{t}$.

Now let us consider the equation (1) again. Since we have $Q^{\prime} Q^{\prime \prime}=Q^{\prime}, Q^{\prime \prime} Q^{\prime}=$ $Q^{\prime \prime}$, it is clear that

$$
\left(Q^{\prime}\right)_{i j}=0, \quad 1 \leqslant i \leqslant s \Leftrightarrow\left(Q^{\prime \prime}\right)_{i j}=0, \quad 1<i<s .
$$

Let $\left\{T, C_{1}, C_{2}, \ldots, C_{p}\right\}$ be the partition (as in Lemma 2) corresponding to the idempotent matrix $Q^{\prime}$. Let $i \in C_{k_{1}}, j \in C_{k_{2}}\left(k_{1} \neq k_{2}\right)$. Then $\left(Q^{\prime}\right)_{i i}>0$ and $\left(Q^{\prime}\right)_{i j}=$ 0 ; also, by equation $(1),\left(Q^{\prime}\right)_{i j} \geqslant\left(Q^{\prime}\right)_{i i}\left(Q^{\prime \prime}\right)_{i j}$ implying that $\left(Q^{\prime \prime}\right)_{i j}=0$ and thus, for $i \notin T$,

$$
\left(Q^{\prime}\right)_{i j}=0 \Leftrightarrow\left(Q^{\prime \prime}\right)_{i j}=0 .
$$

This means that all the limit points of a convergent stochastic chain have the same unique partition (via Lemma 2) associated with them. Thus, we can make the following definition. 
Definition 2. The unique partition that corresponds to all the (idempotent) limit points of a convergent stochastic chain is called the basis of the chain.

For a convergent bistochastic chain, the ' $T$ ' set of the basis is empty. It can be easily verified using Lemma 2 that in case the basis of the chain has its ' $T$ ' set empty, then

$$
Q^{\prime} Q^{\prime \prime}=Q^{\prime} \text { and } Q^{\prime \prime} Q^{\prime}=Q^{\prime \prime} \Rightarrow Q^{\prime}=Q^{\prime \prime}
$$

where $Q^{\prime}$ and $Q^{\prime \prime}$ are as above, and therefore, $\lim _{k \rightarrow \infty} \lim _{n \rightarrow \infty} P_{k, n}$ exists. However, this is not a necessary condition for the limit to exist. The following examples will help us clarify this and also the context of the paper.

EXAMPLE A. The chain given by

$$
P_{n} \equiv\left[\begin{array}{cccc}
a_{n} & \frac{1}{3}-a_{n} & \frac{2}{3}-a_{n} & a_{n} \\
a_{n} & \frac{1}{3}-a_{n} & \frac{2}{3}-a_{n} & a_{n} \\
a_{n} & \frac{1}{3}-a_{n} & \frac{2}{3}-a_{n} & a_{n} \\
0 & 0 & 0 & 1
\end{array}\right], \quad 0<a_{n}<\frac{1}{3},
$$

is a convergent chain for all possible choices of $a_{n}$ between 0 and $\frac{1}{3}$ and if we write $B=\lim _{k} \lim _{n} P_{k, n}$, then $B$ will be a stochastic matrix where each row is $\left(\begin{array}{llll}0 & 0 & 0 & 1\end{array}\right)$ whenever $\Sigma_{n} a_{n}$ diverges, and this limit is

$$
\left[\begin{array}{llll}
0 & \frac{1}{3} & \frac{2}{3} & 0 \\
0 & \frac{1}{3} & \frac{2}{3} & 0 \\
0 & \frac{1}{3} & \frac{2}{3} & 0 \\
0 & 0 & 0 & 1
\end{array}\right]
$$

whenever $\sum_{n} a_{n}$ converges. Note that in the latter case, the basis of the chain is given by $T=\{1\}, C_{1}=\{2,3\}, C_{2}=\{4\}$. Here $T$ is nonempty and still for $i$ in $C_{1}$ and $j$ in $C_{2}, \Sigma_{n}\left(P_{n}\right)_{i j}$ converges. In this paper, we prove that this is always the case in the case when the ' $T$ ' set is empty and conjecture that this is also true when $T$ is nonempty. The interesting nature of this result lies in the fact that the convergence of the chain can be characterized by the strong ergodicity of the normalized $C$-blocks of the chain. (See Theorem 8.)

EXAMPLE B. Consider the chain given by

$$
P_{n} \equiv\left[\begin{array}{ccc}
a_{n} & 2 b_{n} & 1-a_{n}-2 b_{n} \\
1-a_{n}-b_{n} & b_{n} & a_{n} \\
b_{n} & 1-3 b_{n} & 2 b_{n}
\end{array}\right]
$$

where $\Sigma_{n} a_{n}$ converges and $\Sigma_{n} b_{n}$ diverges. Following the classical Bernstein theorem [7, Theorem 4.8, p. 105], we see that this chain is weakly (and therefore, strongly) ergodic. In this case, $\lim _{k} \lim _{n} P_{k, n}$ is a bistochastic matrix where each row is $\left(\frac{1}{3} \frac{1}{3} \frac{1}{3}\right)$. The basis consists of only one $C$-class and yet $\Sigma_{n}\left(P_{n}\right)_{23}$ converges. 
EXAMPLE C. Consider the stochastic chain $\left(R_{n}\right)$ given by

$$
\begin{aligned}
R_{n} & =P \text { for } n \text { even, } \\
& =Q_{n} \text { for } n \text { odd, }
\end{aligned}
$$

where the matrices $P$ and $Q_{n}$ are given by

$$
P=\left|\begin{array}{lll}
0 & 1 & 0 \\
0 & 1 & 0 \\
0 & 0 & 1
\end{array}\right|, \quad Q_{n}=\left|\begin{array}{ccc}
a_{n} & b_{n} & 0 \\
1 / n & 1-1 / n & 0 \\
0 & 0 & 1
\end{array}\right|
$$

( $a_{n}$ and $b_{n}$ are nonnegative numbers with sum one). It is easily verified that $\lim _{n} R_{k, n}=P$ for every $k$. The basis of the chain is given by $T=\{1\}, C_{1}=\{2\}$, $C_{2}=\{3\}$. Notice that $\Sigma_{n}\left(R_{n}\right)_{21}$ is divergent.

All the three examples will be found useful later specially in the context of our remark following Theorem 8 characterizing the $C$-classes (for a convergent chain with no ' $T$ ' in its basis) in terms of divergence of certain series. Regarding examples of convergent stochastic chains with no ' $T$ ', we remark that our Theorem 8 characterizes such chains completely and using this theorem, one can obtain any number of examples of such chains.

Example D. This example has been provided by A. Nakassis. This example shows that our Theorem 8 cannot be extended to the case of nonempty ' $T$ '. Consider the stochastic chain $\left(P_{n}\right)$ given by

$$
P_{n}=\left(\begin{array}{cccc}
0 & 1 / 2 & 1 / 2 & 0 \\
1 / n & 0 & 1-1 / n & 0 \\
1 / n & 1-1 / n & 0 & 0 \\
0 & 0 & 0 & 1
\end{array}\right)
$$

Then it can be verified that this is a convergent chain with basis $T=\{1\}$, $C_{1}=\{2,3\}$ and $C_{2}=\{4\}$. But notice that the normalized $C_{1}$-blocks of $\left(P_{n}\right)$ are not even convergent.

\section{Main results.}

Definition 3. A stochastic $0-1$ matrix $A$ is said to belong to the basis of an idempotent stochastic matrix $B$ if $A_{i j}=0$ whenever $i, j$ belong to two different 'non- $T$ ' members of the basis.

LEMMA 4. If the stochastic 0-1 matrices $A$ and $D$ both belong to the basis of some idempotent stochastic matrix (here ' $T$ ' is empty), then the product $A \cdot D$ also belongs to the same basis.

Proof. The proof is immediate since $(A \cdot D)_{i j}=\sum_{k=1}^{s} A_{i k} D_{k j}$.

LEMma 5. ${ }^{1}$ Suppose that the 0-1 stochastic matrices $A$ and $D$ are such that $B A=B D$, where $B=B^{2}$ is stochastic. Then if the basis of $B$ has ' $T$ ' empty and if $D$ belongs to the basis of $B$, then $A$ also belongs to the basis of $B$.

\footnotetext{
${ }^{1}$ This lemma is of independent interest and not used later.
} 
Proof. Suppose that $A$ does not belong to the basis of $B$. Then there are members $C_{k_{1}}, C_{k_{2}}\left(k_{1} \neq k_{2}\right)$ in the basis of $B$ and $i \in C_{k_{1}}, j \in C_{k_{2}}$ such that $A_{i j}=1$. But then since $B_{i i}>0,(B A)_{i j} \geqslant B_{i i} A_{i j}>0$ and therefore, $(B D)_{i j}>0$. This means that there is an $l$ such that $B_{i l} D_{l j}>0$. This is impossible since $B_{i l}>0 \Rightarrow l \in C_{k_{1}}$ and $D_{l j}>0 \Rightarrow l \in C_{k_{2}}$.

LEMMA 6. Let $B$ be a stochastic idempotent matrix such that its basis has ' $T$ ' empty. Let us define the set $K$ by

$$
K=\{X: X \text { is a stochastic } 0-1 \text { matrix and } X \text { belongs to the basis of } B\} \text {. }
$$

Then $K$ is a subsemigroup of the semigroup $S$ of all $s \times s$-1 stochastic matrices. Moreover, if $X \in K$, then $Y X \in K$ for a stochastic 0-1 matrix $Y$ iff $Y \in K$.

Proof. $K$ is a semigroup by Lemma 4. Suppose now that $Y X \in K, X \in K$ and $Y$ is a 0-1 stochastic matrix. Suppose that $Y \notin K$. Then there are members $C_{k_{1}}, C_{k_{2}}$ $\left(k_{1} \neq k_{2}\right)$ in the basis of $B$ and $i \in C_{k_{1}}, j \in C_{k_{2}}$ such that $Y_{i j}=1$. Now choose $l$ so that $X_{j l}=1$. Since $X \in K, l \in C_{k_{2}}$. But then, $(Y X)_{i l} \geqslant Y_{i j} X_{j l}=1$. This is a contradiction, since $Y X \in K$.

Now we consider a convergent stochastic chain $\left(P_{n}\right)$. Let $\lim _{n \rightarrow \infty} P_{k, n}=Q_{k}$. In what follows, we will assume that the basis of the chain has its ' $T$ ' set empty. Then, as we remarked earlier, $\lim _{k \rightarrow \infty} Q_{k}$ exists. Let $Q=\lim _{k \rightarrow \infty} Q_{k}$. Now we take a probability measure $\mu_{n}$ on $S$, the finite semigroup of all $s \times s$-1 stochastic matrices such that $\mu_{n} \leftrightarrow P_{n}$. We define $K$ by

$$
K=\left\{X: X \in S \text { and } X \text { belongs to the basis of the chain }\left(P_{n}\right)\right\} .
$$

Then we claim the following:

Given $\varepsilon>0$, there exist a positive integer $k_{0}\left(k_{0} \equiv k_{0}(\varepsilon)\right)$ and a positive integer $N$ $(N \equiv N(\varepsilon))$ such that $n \geqslant N \Rightarrow$

$$
\mu_{k_{0, n}}(K)>1-\varepsilon
$$

To prove (2), suppose there are $m$ many elements in $S-K$. Let $Z \in S-K$. Then there are $i \in C_{k_{1}}, j \in C_{k_{2}}$, where $C_{k_{1}}$ and $C_{k_{2}}$ are two different members of the basis of $Q$ such that $Z_{i j}=1$. This means that $\Sigma_{k \in C_{k_{1}}} Z_{i k}=0$. Since $\Sigma_{k \in C_{k_{1}}} Q_{i k}=1$ and $\lim _{r \rightarrow \infty} \lim _{n \rightarrow \infty} P_{r, n}=Q$, we see that there exist positive integers $k_{0}$ and $N$ such that $n \geqslant N$ implies for each $l, 1 \leqslant l \leqslant s$,

$$
\sum_{k}\left(P_{k_{0, n},}\right)_{l k}>1-\frac{\varepsilon}{m}
$$

where the summation is over all those $k$ such that $k$ and $l$ both belong to the same member of the basis. Since $P_{k_{0, n}}=\Sigma_{X \in S} X \mu_{k_{0, n}}(X)$, we have

$$
\sum_{k \in C_{k_{1}}}\left(P_{k_{0}, n}\right)_{i k} \leqslant \sum_{X_{i k}=1} \mu_{k_{0}, n}(X) \leqslant \mu_{k_{0}, n}(S-Z) .
$$

Hence, for any $Z \in S-K, n \geqslant N \Rightarrow \mu_{k_{0}, n}(Z)<\varepsilon / m$. This means that $n \geqslant N \Rightarrow$ $\mu_{k_{0}, n}(S-K)<\varepsilon$, which establishes (2).

Next we claim the following: There exist positive integers $n_{0}, N^{\prime}$ and a $\delta>0$ such that $n \geqslant N^{\prime} \Rightarrow$

$$
\max _{x \in S-K} \mu_{n_{0, n}}\left(K x^{-1}\right)+\delta \leqslant \mu_{n_{0, n}}(K) .
$$


To establish the claim, we suppose that the claim is false. Then for every increasing sequence $\left(k_{i}\right)$ of positive integers, there exists a positive integer $n_{i}^{\prime}>N_{i}\left(N_{i}\right.$ is the integer $N(\varepsilon)$ and $k_{i}$ is $k_{0}(\varepsilon)$ ) in (2) for $\varepsilon=1 / 2^{i}$ and some $x_{i} \in S-K$ such that

$$
\mu_{k_{i}, n_{i}^{\prime}}\left(K x_{i}^{-1}\right)+\frac{1}{2^{i}}>\mu_{k_{i}, n_{i}^{\prime}}(K) \text {. }
$$

Let $x \in S-K$ be such that $x_{i}=x$ for infinitely many $i$. Then by using (2) and taking suitable subsequences $k_{i_{j}}\left(\equiv p_{j}\right.$, say) and $n_{i_{j}}^{\prime}\left(\equiv m_{j}\right.$, say), we have

$$
\lim _{j \rightarrow \infty} \mu_{p_{j}, m_{j}}\left(K x^{-1}\right)=1, \quad x \in S-K \text {. }
$$

Now $\mu_{p_{j}, m_{j}} \leftrightarrow P_{p_{j}, m_{j}}$. Let $\lambda^{\prime}$ be a limit point of the sequence $\left(\mu_{p_{j}, m_{j}}\right)$. Let $\lambda^{\prime} \leftrightarrow M^{\prime}$. Since $P_{l, p_{j}} P_{p_{j}, m_{j}}=P_{l, m_{j}}$, it is clear that $Q_{l} M^{\prime}=Q_{l}$ and so passing $l$ to $\infty$, we have

$$
Q M^{\prime}=Q \text {. }
$$

By (5), $\lambda^{\prime}\left(K x^{-1}\right)=1$. Therefore, we have:

$$
S_{\lambda^{\prime}} \subset K x^{-1} \text {. }
$$

Since $x \notin K$, there are members $C_{k_{1}}, C_{k_{2}}\left(k_{1} \neq k_{2}\right)$ of the basis of the chain and $i \in C_{k_{1}}, j \in C_{k_{2}}$ such that $(x)_{i j}=1$. Now by (6), $0<Q_{i i}=\Sigma_{l} Q_{i l} M_{l i}^{\prime}$ so there exists $l \in C_{k_{1}}$ such that $M_{l i}^{\prime}>0$. Since $\lambda^{\prime} \leftrightarrow M^{\prime}$, there is $y \in S_{\lambda^{\prime}}$ such that $(y)_{l i}=1$. By (7), $y x \in K$. But then, $(y x)_{l j} \geqslant y_{l i} x_{i j}=1$. This is a contradiction since $l \in C_{k_{1}}$ and $j \in C_{k_{2}}$ and $y x \in K$. This establishes our claim (4).

Now we prove the following assertion:

$$
\sum_{n=1}^{\infty} \mu_{n}(S-K)<\infty
$$

Proof of (8). Let $n_{0}$ be as in (4). Write: $A_{n}=\max _{x \in S-K} \mu_{n_{0, n}}\left(K x^{-1}\right)$. Then there exists $\delta>0$ such that $n \geqslant N^{\prime} \Rightarrow A_{n}+\delta \leqslant \mu_{n_{0}, n}(K)$. Now

$$
\begin{aligned}
\mu_{n_{0}, n+1}(K) & =\sum_{x \in S} \mu_{n_{0}, n}\left(K x^{-1}\right) \mu_{n+1}(x) \\
& =\sum_{x \in K} \mu_{n_{0}, n}\left(K x^{-1}\right) \mu_{n+1}(x)+\sum_{x \in S-K} \mu_{n_{0}, n}\left(K x^{-1}\right) \mu_{n+1}(x) \\
& \leqslant \mu_{n_{0}, n}(K) \mu_{n+1}(K)+A_{n} \mu_{n+1}(S-K) \\
& =\mu_{n_{0}, n}(K)\left[1-\mu_{n+1}(S-K)\right]+A_{n} \mu_{n+1}(S-K) \\
& =\mu_{n_{0, n},}(K)-\left[\mu_{n_{0}, n}(K)-A_{n}\right] \mu_{n+1}(S-K) \\
& \leqslant \mu_{n_{0, n},}(K)-\delta \mu_{n+1}(S-K) .
\end{aligned}
$$

Repeating the above procedure, we have

$$
\mu_{k_{0, n+1}}(K) \leqslant \mu_{k_{0,} N^{\prime}}(K)-\delta \sum_{i=N^{\prime}+1}^{n+1} \mu_{i}(S-K)
$$

so that for each $n>N^{\prime}$, we have

$$
\sum_{i=N^{\prime}+1}^{n+1} \mu_{i}(S-K) \leqslant \frac{2}{\delta} .
$$

Thus, (8) is established. 
Now consider $i \in C_{k_{1}}, j \in C_{k_{2}}\left(k_{1} \neq k_{2}\right)$ where $C_{k_{1}}, C_{k_{2}}$ are two different members of the basis of the chain. Since $P_{n} \leftrightarrow \mu_{n}, P_{n}=\Sigma_{x \in S} x \mu_{n}(x)$; therefore,

$$
\left(P_{n}\right)_{i j} \leqslant \sum_{x_{i j}=1} \mu_{n}(x) \leqslant \mu_{n}(S-K)
$$

which implies that $\sum_{n=1}^{\infty}\left(P_{n}\right)_{i j}<\infty$, by (8). Thus we have proven the crucial result of our paper.

THEOREM 7. Let $\left(P_{n}\right)$ be a convergent stochastic chain with the basis $\left\{C_{1}, C_{2}, \ldots, C_{p}\right\}$. Then for $i, j$ belonging to two different $C_{k}$ 's $\sum_{n=1}^{\infty}\left(P_{n}\right)_{i j}<\infty$.

Now we consider a convergent stochastic chain $\left(P_{n}\right)$ with the basis $\left\{C_{1}, C_{2}, \ldots, C_{p}\right\}$. Let us define for each $i, 1 \leqslant i \leqslant p$, and for each $n \geqslant 1$ an $n\left(C_{i}\right) \times n\left(C_{i}\right)$ matrix as follows:

$$
\left(P_{n}\left(C_{i}\right)\right)_{j k}=\frac{\left(P_{n}\right)_{j k}}{\sum_{l \in C_{i}}\left(P_{n}\right)_{j l}}, \quad j \in C_{i}, k \in C_{i} .
$$

Write $Q_{i, n} \equiv P_{n}\left(C_{i}\right)$. Then $\left(Q_{i, n}\right)_{n>1}$ is a stochastic chain with $n\left(C_{i}\right)$ states. Now if we define the chain $\left(P_{n}^{\prime}\right)$ with $s$ states by

$$
\begin{aligned}
\left(P_{n}^{\prime}\right)_{j k} & =\left(Q_{i n}\right)_{j k}, \quad \text { if } j, k \text { both belong to } C_{i}, \\
& =0, \text { if } j, k \text { belong to two different } C_{i}^{\prime} \text { s, }
\end{aligned}
$$

then the chain $\left(P_{n}^{\prime}\right)$ is equivalent to the convergent chain $\left(P_{n}\right)$ (because of Theorem 7) in the following sense:

$$
\sum_{n=1}^{\infty}\left|\left(P_{n}\right)_{j k}-\left(P_{n}^{\prime}\right)_{j k}\right|<\infty
$$

for each $j, k(1 \leqslant j, k \leqslant s)$. It is now easy to show that the chain $\left(P_{n}^{\prime}\right)$ is also convergent with the same basis $\left\{C_{1}, C_{2}, \ldots, C_{p}\right\}$. It follows that for each $i$ $(1 \leqslant i \leqslant p)$, the chain $\left(P_{n}\left(C_{i}\right)\right)$ is strongly ergodic (i.e., for each $k \geqslant 1$, the products $P_{k, n}\left(C_{i}\right) \equiv P_{k+1}\left(C_{i}\right) \ldots P_{n}\left(C_{i}\right)$ converge to a stochastic matrix with identical rows). Now it is not difficult to see that the following theorem is true.

THEOREM 8. Let $\left(P_{n}\right)$ be a convergent stochastic chain with basis $\left\{C_{1}, C_{2}, \ldots, C_{p}\right\}$. Then (i) for $j, k$ belonging to two different $C_{i}^{\prime} s, \sum_{n=1}^{\infty}\left(P_{n}\right)_{j k}<\infty$ and (ii) the chains $\left(P_{n}\left(C_{i}\right)\right)$ for each $i$ are each strongly ergodic with no ' $T$ ' class. Conversely, the conditions (i) and (ii) are also sufficient for the convergence of the chain $\left(P_{n}\right)$ with basis $\left\{C_{1}, C_{2}, \ldots, C_{p}\right\}$.

This theorem can be useful for practical reasons in the sense that convergence of larger chains can be checked by checking strong ergodicity for the corresponding smaller chains. It is also clear that any convergent stochastic chain whose basis has the ' $T$ ' set empty is strongly ergodic if for some $j$ and each $i, \sum_{n=1}^{\infty}\left(P_{n}\right)_{i j}=\infty$. In particular, any convergent bistochastic chain for which this last divergence condition holds is strongly ergodic.

$A$ characterization of the $C$-classes. Two states $t$ and $j$ are in different $C$-classes iff for every sequence $\left(a_{k}\right)$ of positive integers, the series $\sum_{k=1}^{\infty}\left(P_{a_{k}, a_{k+1}}\right)_{t j}$ is convergent. 
To see this, we first observe that considering

$$
\mu_{n_{0}, a_{n+1}}(K)=\sum_{x \in S} \mu_{n_{0}, a_{n}}\left(K x^{-1}\right) \mu_{a_{n}, a_{n+1}}(x)
$$

and following the proof of (8) almost identically, the nontrivial part (the only if part) of the above statement follows easily. To check the validity of the "if" part, notice that for $t, j$ in the same $C$-class, $Q_{t j}>0$ and there exist sequences $\left(k_{i}\right)$ and $\left(n_{k_{i}}\right)$ of positive integers such that for $n \geqslant n_{k_{i}},\left(P_{k_{i}, n}\right)_{t j}>\frac{1}{2} Q_{t j}>0$. We can now construct the sequence $m(i)$ such that $k_{m(i+1)}>n_{k_{m(i)}}$ for each $i$. Write $p_{i}=k_{m(i)}$. Then it is clear that

$$
\sum_{i=1}^{\infty}\left(P_{p_{i} P_{i+1}}\right)_{t j}=\infty
$$

The proof of the characterization is now complete.

4. An application. We will now present an application of Theorem 7 to a problem on probability measures on semigroups. It is well known that if $\left(\mu_{n}\right)$ is a sequence of probability measures on a finite group $S$ such that $\lim _{n \rightarrow \infty} \mu_{k, n}=\lambda_{k}$ exists and $\lim _{k \rightarrow \infty} \lambda_{k}=\lambda_{\infty}$ exists, then $\sum_{n=1}^{\infty} \mu_{n}\left(S-S_{\lambda_{\infty}}\right)<\infty$. In other words, the measures $\mu_{n}$ can, for most purposes, be considered to be concentrated on $S_{\lambda_{\infty}}$. This result is not true on finite semigroups $S$. For example, take $S=\{0,1\}$ with multiplication and $\mu_{n} \equiv \mu$ where $\mu(0)=\mu(1)=\frac{1}{2}$. Then $\lim _{n \rightarrow \infty} \mu^{n}=$ the point mass at 0 , but $\mu(1)>0$. The following theorem, as an application of Theorem 7 , will give us the information with respect to the above behavior in semigroups. We will use another type of correspondence, originally due to P. Martin-Löf.

TheOREM 9. Suppose that $S=\left\{a_{1}, a_{2}, \ldots, a_{s}\right\}$ is a finite semigroup with an identity $e \equiv a_{u}$. Let $\mu_{n} \in P(S)$. Suppose also that

$$
\left.\lim _{n} \mu_{k, n}=\lambda_{k}, \quad \lim _{k} \lambda_{k}=\lambda_{\infty} \text { and } e \in S_{\lambda_{\infty}} \equiv H \text { (the support of } \lambda_{\infty}\right) \text {. }
$$

Then $\Sigma_{n} \mu_{n}(S-H)$ converges.

[For each $a_{i}$ in $S$, we define the 0-1 matrix $(s \times s) A_{i}$ by

$$
\begin{aligned}
\left(A_{i}\right)_{j k} & =1, & & \text { if } a_{j} a_{i}=a_{k}, \\
& =0, & & \text { otherwise. }
\end{aligned}
$$

Then the mapping $\Phi: S \rightarrow S^{\prime} \equiv$ the semigroup of stochastic matrices of order $n(S)$ defined by $\Phi\left(a_{i}\right)=A_{i}$ is an isomorphism. For $\mu \in P(S)$, define $P_{\mu} \in S^{\prime}$ by $P_{\mu}=\Sigma_{x \in S} \Phi(x) \mu(x)$. Then $\left(P_{\mu}\right)_{j k}=\mu\left(a_{j}^{-1} a_{k}\right)$. It can be verified that the correspondence $\mu \rightarrow P_{\mu}$ is a homomorphism from $P(S)$ into $S^{\prime}$.]

Proof. We have $S=S \cdot H$. Let $\mu_{n} \rightarrow P_{n}, \lambda_{k} \rightarrow Q_{k}$ and $\lambda_{\infty} \rightarrow Q$ as discussed above. Then for each $j$, there exists $i$ such that $a_{i}^{-1} a_{j} \cap H \neq \varnothing$ so that $\lambda_{\infty}\left(a_{i}^{-1} a_{j}\right)>$ 0 ; then

$$
\left(Q_{\infty}\right)_{i j}>0 \text {. }
$$

Clearly, $\lim _{n \rightarrow \infty} P_{k, n}=Q_{k}$ and $\lim _{k \rightarrow \infty} Q_{k}=Q_{\infty}$. Hence, by (9), the basis of the convergent chain has its ' $T$ ' set empty. Let the basis be $\left\{C_{1}, C_{2}, \ldots, C_{p}\right\}$. Let $S_{i}=\left\{a_{j} \mid j \in C_{i}\right\}$; then $S=S_{1} \cup S_{2} \cup \ldots \cup S_{p}$ is a partition of $S$. Note that 
$g \in H \Rightarrow \lambda_{\infty}(g)>0$. If $S_{i} g \cap S_{j} \neq \varnothing$ (for some $i, j, i \neq j$ ), then $g \in a_{k}^{-1} a_{l}$ for some $a_{k} \in S_{i}$ and $a_{l} \in S_{j}$, so that $k \in C_{i}$ and $l \in C_{j}(i \neq j)$. Then $\left(Q_{\infty}\right)_{k l}=0$; but $\left(Q_{\infty}\right)_{k l}=\lambda_{\infty}\left(a_{k}^{-1} a_{l}\right)>0$, a contradiction. Hence, $g \in H \Rightarrow S_{i} g \subset S_{i}(1<i<p)$. Let $H_{1}=\left\{g \in S: S_{i} g \subset S_{i}, 1 \leqslant i \leqslant p\right\}$. Suppose that $a_{u} \equiv e \in S_{t}$. If $h \in H_{1}$, then $S_{t} \cdot h \subset S_{t}$ and therefore, $h \in S_{t}$. If $h \equiv a_{m}$, then $m$ and $u$ both belong to $C_{t}$ so that $\left(Q_{\infty}\right)_{u m}>0$; therefore, $\lambda_{\infty}\left(a_{u}^{-1} a_{m}\right) \equiv \lambda_{\infty}(h)>0 \Rightarrow h \in H$. Hence, $H_{1} \subset H$. Now $g \in S-H \Rightarrow$

$\exists i, j(i \neq j)$ such that $S_{i} g \cap S_{j} \neq \varnothing$, and therefore, $g \in a_{k_{1}}^{-1} a_{k_{2}}$, where $k_{1} \in C_{i}, k_{2} \in C_{j}$.

By Theorem 7,

$$
\begin{aligned}
\sum_{n=1}^{\infty}\left(P_{n}\right)_{k_{1}, k_{2}} & <\infty \Rightarrow \sum_{n=1}^{\infty} \mu_{n}\left(a_{k_{1}}^{-1} a_{k_{2}}\right)<\infty \\
& \Rightarrow \sum_{n=1}^{\infty} \mu_{n}(g)<\infty .
\end{aligned}
$$

Since $S$ is finite, the theorem follows.

The above theorem shows that it is possible to obtain limit theorems for probability measures on semigroups and groups from limit theorems for Markov chains. Below we present another result (omitting the proof) in this direction using the well-known Bernstein condition for weak ergodicity [7, p. 105] for a nonhomogeneous Markov chain.

TheOREM 10. Let $\mu_{n} \in P(G), n \geqslant 1$, where $G$ is a finite group. Suppose that $\sum_{n=1}^{\infty}\left\{\min \mu_{n}(x)\right\}=\infty$. Then for each $k \geqslant 1, \mu_{k, n} \rightarrow \lambda=\lambda^{2} \in P(G)$.

5. Nonnegative idempotent matrices. Here we show that a basis similar to that for stochastic idempotent matrices exists for nonnegative idempotent matrices and then present explicitly the structure of such a matrix and other related results in details. The reason for including this section is that convergence problems for products of nonnegative matrices appear very naturally in various problems in demography and, as in the stochastic case, the role of idempotents can be important in many contexts. (This question will be dealt with in details elsewhere.) Also, determination of the structure of nonnegative idempotents seems to be more difficult than the corresponding stochastic problem, and finally, as far as we know this result is missing in the literature.

Our result can be described by the following theorem.

THEOREM 11. Let $P \equiv\left(P_{i j}\right)$ be an $s \times s$ nonnegative idempotent matrix. Then there is a partition of $\{1,2, \ldots, s\}$ into classes $\left\{T, C_{1}, C_{2}, \ldots, C_{p}\right\}$ such that the following results hold:

(i) the jth row or the jth column or both consist of only zeros iff $j \in T$;

(ii) $P_{i j}=0$ whenever $i \in C_{k_{1}}$ and $j \in C_{k_{2}}\left(k_{1} \neq k_{2}\right)$;

(iii) in any $C_{k}$-block $(1 \leqslant k \leqslant p)$ of the matrix, the rows are all proportional, each entry is positive, and the sum of the diagonal entries is 1; 
(iv) if $i \in T$ and the ith column is a zero column, then for $j, k$ in $C_{t}(1<t<p)$, $P_{i j} / P_{i j}=P_{i k} / P_{j k}$

(v) if $j \in T$ and the jth row is a zero row, then for $i, k$ in $C_{t}(1<t<p)$, $\boldsymbol{P}_{i j} / \boldsymbol{P}_{i i}=\boldsymbol{P}_{k j} / \boldsymbol{P}_{k i}$.

In particular, if $P$ has no zero rows or zero columns, then $P$ must be of the form

$$
\left(\begin{array}{ccccc}
C_{1} & 0 & 0 & \cdots & 0 \\
0 & C_{2} & 0 & \cdots & 0 \\
0 & 0 & C_{3} & \cdots & 0 \\
\vdots & \vdots & \vdots & & \vdots \\
0 & 0 & 0 & & C_{p}
\end{array}\right)
$$

where the $C_{k}$-blocks are as described in (iii) above.

Proof. Let us first suppose that the theorem holds for any nonnegative idempotent matrix which has no zero rows or zero columns. We then claim that the theorem also holds in the general case. To see this, let $T$ be the set $\{j$ : either $P_{i j}=0$ for every $i$ or $P_{j i}=0$ for every $i$ ) and let $T$ be nonempty. Then it is easy to see that $Q$, the restriction of $P$ to the complement of $T$, is a nonnegative idempotent matrix with no zero rows or zero columns. Then by our above assumption, there is a partition of the complement of $T$ into classes $\left\{C_{1}, C_{2}, \ldots, C_{p}\right\}$ such that the $C_{k}$-blocks of $P$ satisfy properties (ii) and (iii) in the theorem. Now if $i \in T$ and the $i$ th column of $P$ is a zero column, then for $j, k$ in $C_{t}$ $(1<t<p)$, we have

$$
P_{i j}=\sum_{u \in C_{t}} P_{i u} P_{u j} \text { and } P_{i k}=\sum_{u \in C_{t}} P_{i u} P_{u k}
$$

Notice that $P_{u j}>0$ for each $u$ in $C_{t}$ and therefore, $P_{i j}=0$ iff $P_{i k}=0$. In case both $\boldsymbol{P}_{i j}$ and $\boldsymbol{P}_{i k}$ are positive, then since the rows of $C_{t}$-blocks of $\boldsymbol{P}$ are proportional (by property (iii)), we have $P_{i j} / P_{j k}=P_{u j} / P_{u k}$ (for each $u$ in $C_{t}$, and therefore) = $\boldsymbol{P}_{i j} / \boldsymbol{P}_{i k}$. Thus property (iv) (and similarly, property (v)) follows and our claim is verified. This means that with no loss of generality we can assume that $\boldsymbol{P}$ has no zero rows or zero columns. To prove the theorem, we will use induction on the dimension $s$ of $P$.

The theorem is easily verified when $s=1$ or $s=2$. So let us assume that the theorem is valid for all nonnegative idempotent matrices of dimension less than $s$. To prove the theorem for dimension $s$, we use the following steps.

Step I. In this step, we show that $P_{i i}>0$ for each $i, 1<i<s$.

Proof of STEP I. Our proof is by contradiction. Suppose that $P_{11}=0$. Since $P_{11}=\sum_{j=1}^{s} P_{1 j} P_{j 1}$, we have

$$
P_{1 j} P_{j 1}=0 \text { for each } j
$$

Let us define the set $A$ by

$$
A=\left\{i: P_{1 i}>0\right\} \text {. }
$$


Then $A$ as well as its complement is nonempty. Notice that for $i \notin A, 0=P_{1 i}=$ $\sum_{j \in A} P_{1 j} P_{j i}$ and therefore,

$$
P_{j i}=0 \quad \text { whenever } j \in A \text { and } i \notin A \text {. }
$$

Using (12), it can be easily verified that the matrices $P_{1}$ and $P_{2}$, the restrictions of $P$ to the sets $A$ and the complement of $A$ respectively, are both idempotent matrices of dimension less than $s$. Then $P$ looks like

$$
\left(\begin{array}{l|l}
P_{2} & \\
\hline 0 & P_{1}
\end{array}\right)
$$

Now following the induction hypothesis, we can write the basis for $P_{1}$ as: $\left\{T^{\prime}, C_{1}^{\prime}, C_{2}^{\prime}, \ldots, C_{m}^{\prime}\right\}$. Choose $i \notin A, i \neq 1$ such that $P_{i 1}>0$. (Since $P$ has no zero column, there exists such an $i$.) Let us write $C_{1}^{\prime}=\left\{j_{1}, j_{2}, \ldots, j_{r}\right\}$. Let $\beta_{1}, \beta_{2}, \ldots, \beta_{r}$ be positive real numbers such that the $t\left(\right.$ th) row of the $C_{1}^{\prime}$-block of $P_{1}$ is $\beta_{t}$-times the $I$ st row of this block so that

$$
\sum_{t=1}^{r} \beta_{t} P_{j_{k} j_{t}}=\beta_{k}, \quad 1 \leqslant k \leqslant r
$$

Also we have

$$
\begin{aligned}
P_{i j t} & =\sum_{k \in A} P_{i k} P_{k j_{t}}+\sum_{k \notin A} P_{i k} P_{k j_{t}} \\
& \geqslant \sum_{k \in C_{i}^{\prime}} P_{i k} P_{k j_{t}}+\sum_{k \notin A} P_{i k} P_{k j} .
\end{aligned}
$$

Hence,

$$
\sum_{t=1}^{r} \beta_{t} P_{i j t} \geqslant \sum_{k \in C_{i}^{\prime}} P_{i k}\left(\sum_{t=1}^{r} \beta_{t} P_{k j_{t}}\right)+\sum_{k \notin A} P_{i k}\left(\sum_{t=1}^{r} \beta_{t} P_{k j_{t}}\right) .
$$

Now by (13), the first sum on the right is equal to the sum on the left; as a result, the second sum on the right must be zero and therefore since $P_{i 1}>0$ and $1 \notin A$, we must have $\Sigma_{t=1}^{r} \beta_{t} P_{1 j_{t}}=0$. Since for each $t, \beta_{t}>0, P_{1 j_{t}}=0$ for each $t$. This contradicts (11). Thus, Step I is verified.

Step II. In this step, we show that $P_{i j}=0$ iff $P_{j i}=0$.

Proof OF STEP II. For simplicity in writing, we show that $P_{12}=0$ implies $P_{21}=0$. So let $P_{12}=0$. Let $D=\left\{k: P_{1 k}>0\right\}$. Then $D$ as well as its complement is nonempty. (In fact, $1 \in D$ and $2 \notin D$.) Now for $k \notin D, 0=P_{1 k}=\Sigma_{j \in D} P_{1 j} P_{j k}$ and therefore,

$$
P_{j k}=0 \quad \text { whenever } j \in D \text { and } k \notin D .
$$

This means that the matrices $P_{3}$ and $P_{4}$, the restrictions of $P$ on $D$ and its complement respectively, are both idempotent matrices of dimension less than $s$.

Let us now write $C^{\prime}=\left\{j_{0}, j_{1}, \ldots, j_{r}\right\}, j_{0}=1$, where $C^{\prime}$ is the $C$-class in $D$ for the matrix $P_{3}$ that contains 1 . (Note that by induction hypothesis, the theorem is valid for the matrices $P_{3}$ and $P_{4}$.) Let $\beta_{0}, \beta_{1}, \ldots, \beta_{r}$ be positive reals such that the $t$ th row in the $C^{\prime}$-block of $P_{3}$ is $\beta_{t}$-times the $I$ st row in this block. Then we have 


$$
\begin{aligned}
\sum_{t=0}^{r} \beta_{t} P_{2 j_{t}} & =\sum_{k} P_{2 k}\left(\sum_{t=0}^{r} \beta_{t} P_{k j_{t}}\right) \\
& \geqslant \sum_{k \in C^{\prime}}+\sum_{k \notin D} \\
& =\sum_{t=0}^{r} \beta_{t} P_{2 j_{t}}+\sum_{k \notin D} P_{2 k}\left(\sum_{t=0}^{r} \beta_{t} P_{k j_{t}}\right)
\end{aligned}
$$

since $\sum_{t=0}^{r} \beta_{t} P_{k j_{t}}=\beta_{m}$ for $k=j_{m}$ in $C^{\prime}$ (this follows easily by induction hypothesis and property (iii)). It now follows that the second sum on the right side of the above inequality is zero and here taking 2 for $k$ and $t=0$, we have $P_{21}=0$. Thus, Step II is verified.

Step III. In this step we complete the proof of the theorem. Choose any $i$, $1 \leqslant i \leqslant s$, and let $C_{i}=\left\{j: P_{i j}>0\right\}$. For any $j, k$ in $C_{i}, P_{j k} \geqslant P_{j i} P_{i k}>0$. Also, if $j \in C_{i}$ and $k \notin C_{i}$, then $P_{j k}=P_{k j}=0$ since $P_{i j} P_{j k} \leqslant P_{i k}=0$. This means that $Q_{i}$, the restriction of $P$ to $C_{i}$, is a positive idempotent matrix. Therefore, the proof of the theorem will be complete if we show that every positive idempotent matrix must be of rank 1 . To this end, let $Q \equiv\left(Q_{i j}\right)$ be such a matrix. Let $a(i, j, k)$ be positive reals such that $Q_{i j}=a(i, j, k) \cdot Q_{k j}$. Choose $j_{i k}$ such that

$$
a\left(i, j_{i k}, k\right)=\max \{a(i, j, k): 1 \leqslant j \leqslant s\} \text {. }
$$

Now we have

$$
a\left(i, j_{i k}, k\right) \sum_{t} Q_{k t} Q_{t_{j k}}=Q_{j_{j k}}=\sum_{t} a(i, t, k) Q_{k t} Q_{t j_{i k}}
$$

Since $Q$ is positive, it is clear that $a(i, t, k)=a\left(i, j_{i k}, k\right)$ for each $t$. This means that the $i$ th and the $k$ th rows of $Q$ are proportional. Hence, $\operatorname{rank}(Q)=1$.

Before we close this paper, it is relevant to consider the consequences of equation (1) for nonnegative idempotent matrices. In Theorem 11, the partition $\left\{T, C_{1}, C_{2}, \ldots, C_{p}\right\}$ for the matrix $P$ will be called its basis. Furthermore, $T_{r}$ and $T_{c}$ will denote subsets of $T$ such that

$$
\begin{aligned}
& T_{r}=\left\{t \in T: P_{t i}=0 \text { for each } i\right\}, \\
& T_{c}=\left\{t \in T: P_{i t}=0 \text { for each } i\right\} .
\end{aligned}
$$

Now let $Q$ and $Q^{\prime}$ be two nonnegative idempotent matrices such that $Q Q^{\prime}=Q$ and $Q^{\prime} Q=Q^{\prime}$. Suppose that the bases of $Q$ and $Q^{\prime}$ are respectively

$$
\begin{array}{r}
Q:\left\{T_{r}, T_{c}, C_{1}, C_{2}, \ldots, C_{p}\right\}, \\
Q^{\prime}:\left\{T_{r}^{\prime}, T_{c}^{\prime}, C_{1}^{\prime}, C_{2}^{\prime}, \ldots, C_{q}^{\prime}\right\} .
\end{array}
$$

Note that $T_{r}$ and $T_{c}$ need not be disjoint, whereas the $C_{i}$ 's are pairwise disjoint. Let us consider some examples:

(i) Let

$$
Q=\left(\begin{array}{lll}
0 & 1 & 1 \\
0 & 0 & 0 \\
0 & 1 & 1
\end{array}\right) \text { and } Q^{\prime}=\left(\begin{array}{lll}
0 & 1 & 1 \\
0 & 1 & 1 \\
0 & 0 & 0
\end{array}\right)
$$


Then $Q$ and $Q^{\prime}$ are both idempotent, $Q Q^{\prime}=Q$, and $Q^{\prime} Q=Q^{\prime}$. Also, $T_{r}=\{2\}$, $T_{c}=\{1\}$, and $C_{1}=\{3\}, T_{r}^{\prime}=\{3\}, T_{c}^{\prime}=\{1\}$, and $C_{1}^{\prime}=\{2\}$.

(ii) Let

$$
Q=\left(\begin{array}{lll}
0 & 1 & 1 \\
0 & 0 & 0 \\
0 & 1 & 1
\end{array}\right) \text { and } Q^{\prime}=\left(\begin{array}{lll}
0 & a & a \\
0 & b & b \\
0 & b & b
\end{array}\right) \text {, }
$$

where $b=\frac{1}{2}$ and $a>0$. Then $Q$ and $Q^{\prime}$ are both idempotent, $Q Q^{\prime}=Q$, and $Q^{\prime} Q=Q^{\prime}$. Also, $T_{c}^{\prime}=\{1\}$ and $C_{1}^{\prime}=\{2,3\}$.

Now we consider the consequences of the equations $Q Q^{\prime}=Q$ and $Q^{\prime} Q=Q^{\prime}$ where $Q$ and $Q^{\prime}$ are as above in (15). We claim the following results:

(I) $T_{c}=T_{c}^{\prime}$ and $p=q$;

(II) for $i$ and $j$ in two different $C$-classes in the basis of $Q^{\prime}, Q_{i j}=0$;

(III) for each $i(1 \leqslant i \leqslant p)$, there is a $j(i)$ such that $C_{i}^{\prime} \subset C_{j(i)} \cup A_{i}$, where $A_{i}$ is either empty or a subset of $T_{r}$.

In (II) and (III), dual results are, of course, also valid.

Proof. Clearly $T_{c}=T_{c}^{\prime}$ (because of the way matrices multiply). Also, $p=$ $\operatorname{rank}(Q)=\operatorname{rank}\left(Q^{\prime}\right)=q$. If $i \in C_{1}^{\prime}$ and $j \in C_{2}^{\prime}$, then

$$
0=Q_{i j}^{\prime}=\sum_{t} Q_{i t}^{\prime} Q_{i j}
$$

Since for $t \in C_{1}^{\prime}, Q_{i t}^{\prime}>0, Q_{t j}=0$ for each $t \in C_{1}^{\prime}$. This proves (II). To prove (III), let $Q^{(i)}$ and $Q^{(i)}$ be respectively the restrictions of $Q$ and $Q^{\prime}$ to $C_{i}$. Then it is easy to verify that $Q^{(i)}=Q^{\prime(i)} \cdot Q^{(i)}$. Since $\operatorname{rank}\left(Q^{(i)}\right)$ is 1 , the rank of $Q^{(i)}$ is at most 1 . Now notice that $C_{i}^{\prime}$ cannot intersect more than one $C$-class in the basis of $Q$. For, suppose that $k \in C_{i}^{\prime} \cap C_{1}$ and $t \in C_{i}^{\prime} \cap C_{2}$; then, $Q_{k k}>0, Q_{k t}=Q_{t k}=0$, and $Q_{t t}>0$ and therefore, the restriction of $Q$ to $C_{i}^{\prime}$ has rank more than 1 , which contradicts our earlier result. Thus, (III) follows.

ACKNowledgement. The author is thankful to Dean Isaacson for pointing out an error in an example in an earlier draft of this paper. Edwin Clark has also kindly informed the author, after the paper was accepted for publication, that a result similar to our Theorem 11 appears in the LSU thesis of D. Brown; our proof of this theorem is, however, quite different. Our proof is elementary and does not use Frobenius' theorem or any structure theorem based on nilpotence and similarity. The author is also thankful to J. Wolfowitz for many stimulating conversations on the subject of this paper.

\section{BIBLIOGRAPHY}

1. K. L. Chung, Markov chains with stationary transition probabilities, Springer-Verlag, New York, 1967.

2. H. Heyer, Probability measures on locally compact groups, Springer-Verlag, New York, 1977.

3. D. Isaacson and R. Madsen, Markov chains: theory and applications, Wiley, New York, 1976.

4. V. M. Maksimov, Convergence of non-homogeneous bistochastic Markov chains, Theor. Probability Appl. 15 (1970), 604-618.

5. P. Martin-Lö, Probability theory on discrete semigroups, Z. Wahrscheinlichkeitstheorie und Verw. Gebiete 4 (1965), 78-102. 
6. A. Mukherjea, Limit theorems: Stochastic matrices, ergodic Markov chains, and measures on semigroups, Probabilistic Analysis and Related Topics, Vol. 2, Academic Press, New York, 1979, pp. 143-203.

7. E. Seneta, Nonnegative matrices, Wiley, New York, 1973.

8. On the historical development of the theory of finite inhomogeneous Markov chains, Proc. Cambridge Philos. Soc. 74 (1973), 507-513.

9. J. Wolfowitz, Products of indecomposable, aperiodic, stochastic matrices, Proc. Amer. Math. Soc. 14 (1963), 733-737.

Department of Mathematics, University of SOUth Florida, TAMPA, Florida 33620 